\title{
Factors Associated with COVID-19 Mitigation Behavior among US Adults
}

\author{
Debra C. Lemke, PhD (1) \\ Klaus W. Lemke, PhD (2)
}

Affiliations:

1. McDaniel College, Department of Sociology, Westminster, Maryland, USA

2. The Johns Hopkins University Bloomberg School of Public Health, Department of Health Policy and Management, Center for Population Health Information Technology, Baltimore, Maryland, USA

Email addresses:

DL: dlemke@mcdaniel.edu

KL:klemke1@jhu.edu

Corresponding author:

Klaus W. Lemke, PhD

Center for Population Health Information Technology

Department of Health Policy and Management

Johns Hopkins Bloomberg School of Public Health

624 North Broadway / Room 601

Baltimore, MD 21205

Phone: 410 614-4020

Fax: 410 955-0470

Word count: 860 
medRxiv preprint doi: https://doi.org/10.1101/2020.07.20.20157925; this version posted July 25, 2020. The copyright holder for this preprint (which was not certified by peer review) is the author/funder, who has granted medRxiv a license to display the preprint in perpetuity.

It is made available under a CC-BY 4.0 International license.

\begin{abstract}
In early 2020, the CDC issued guidelines for personal COVID-19 mitigation behavior, such as mask-wearing, hand-washing, and social-distancing. We examine individual socio-behavioral factors that potentially predict mitigation compliance using public data. Our analysis finds that pandemic prompted strong mitigation behavior by adults, especially among females, non-whites, urban dwellers, and the psychological unwell. Other positive predictors were post-secondary education and higher income. Health symptoms and clinical risk factors did not predict increased mitigation practices, nor did age 65+ and proximity to infected persons. Our study findings are congruent with a report that pointed to a lack of increased pandemic mitigation practices in households with confirmed infections and health risks. We also point to lower levels of psychological resilience, lower socio-economic status, and nonurban location as potential explanatory factors for lack of mitigation behavior. Understanding what factors are associated with mitigation behavior will be important for policy makers in their efforts to curb the COVID-19 pandemic.
\end{abstract}

\title{
Background
}

In January 2020, the US declared the coronavirus outbreak a public health emergency and subsequently the CDC issued guidelines for personal mitigation behavior, such as mask-wearing, handwashing, and social-distancing.

\section{Objective}

We examine individual socio-economic factors that potentially predict mitigation compliance using public data. ${ }^{1}$ We hypothesize that health risk factors, presence of symptoms, and psychological wellbeing predict mitigation behavior. Understanding what factors are associated with mitigation behavior will be important for policy makers in their efforts to curb the COVID-19 pandemic. 
medRxiv preprint doi: https://doi.org/10.1101/2020.07.20.20157925; this version posted July 25, 2020. The copyright holder for this preprint (which was not certified by peer review) is the author/funder, who has granted medRxiv a license to display the preprint in perpetuity.

It is made available under a CC-BY 4.0 International license .

\section{Methods and Findings}

We used public data from the COVID Impact Survey (CIS), conducted by NORC at the University of Chicago for the Data Foundation. ${ }^{1}$ Several CIS questions were taken from prior national surveys including the American Community Survey, Behavioral Risk Factor Survey, and Current Population Survey. ${ }^{2}$ CIS data for samples of adults aged 18 and older were collected between late April and early June 2020. NORC provided sampling weights.

We combined questionnaire items into a set of parsimonious summary scales and scores. A mitigation compliance scale summed 17 behavior items; several were used previously by the CDC for the 2009 National H1N1 Flu survey. Our summary symptoms score used 8 self-reported symptoms: fever, chills, shortness of breath, cough, sore throat, fatigue or tiredness, muscle pain, and loss of taste and smell. These symptoms were widely recognized indicators for potential SARS-CoV-2 infection. The CDC further identified risk factors for severe COVID-19 illness. ${ }^{3}$ Our risk factor score counted selfreported diabetes, high blood pressure or hypertension, heart disease or stroke, asthma, chronic lung disease or COPD, bronchitis or emphysema, liver disease, cancer, and immunocompromised condition. Lastly, we aggregated CIS items on experiencing feelings of depression, anxiety, hopelessness, loneliness, and stress due to the coronavirus crisis within the last 7 days into an anxiety-depressionstress scale for this study.

Our objective was to develop models to predict mitigation behavior. A base model regressed the mitigation compliance scale on symptom and risk factor scores. Successive models added independent variables including the anxiety-depression-stress scale and dichotomous variables for age 65 and older, individual characteristics (female gender, non-white race, post-secondary degree), household income greater than \$50,000, urban location, and household member has COVID-19. We performed our analyses in SPSS version 26. 
medRxiv preprint doi: https://doi.org/10.1101/2020.07.20.20157925; this version posted July 25, 2020. The copyright holder for this preprint (which was not certified by peer review) is the author/funder, who has granted medRxiv a license to display the preprint in perpetuity.

It is made available under a CC-BY 4.0 International license.

Nearly all adults (99\%) in the survey reported mitigation behavior. The median number of protective practices was 8 (range=17). The mitigation compliance scale reliability was greater than 0.72 and increased to 0.80 in wave 3. Respondents reported 1.0-1.2 (SD=1.4) symptoms on average, and 4952\% were symptomatic. Nearly half (45-49\%) also reported at least 1 risk factor (range=9) consistent with BRFSS estimates. ${ }^{4}$ CIS percentages for cancer, chronic lung disease, diabetes, heart disease, hypertension, and liver disease were within 3 percentage points of BRFSS benchmarks; prevalence of asthma, bronchitis or emphysema, and immunocompromised condition were higher in the CIS. Percentages of depression, anxiety, feelings of hopeless, and loneliness were greater than 35\% in each wave. The anxiety-depression-stress scale (median=7; range=15) reliability was 0.81-0.86 (Table 1).

Regression results for models to predict mitigation behavior are shown in Table 2. Greater anxiety-depression-stress, female gender, post-secondary degree, household income above $\$ 50,000$, and urban location were consistently predictive of more mitigation practices; however, symptoms, risk factors, and age 65+ were not significant predictors across CIS waves. Having a household member with COVID-19 diagnosis was predictive of fewer mitigation practices in wave 3.

\section{Discussion}

The pandemic prompted strong mitigation behavior by adults, especially among females, nonwhites, urban dwellers, and the psychological unwell. Other positive predictors were post-secondary education and higher income. Health symptoms and clinical risk factors did not predict increased mitigation practices, nor did age 65+ and proximity to infected persons. Our study findings are congruent with a report that pointed to a lack of increased pandemic mitigation practices in households with confirmed infections and health risks. ${ }^{1}$ Our results also point to lower levels of psychological resilience, lower socio-economic status, and non-urban location as potential explanatory factors for lack of mitigation behavior. 
medRxiv preprint doi: https://doi.org/10.1101/2020.07.20.20157925; this version posted July 25, 2020. The copyright holder for this preprint (which was not certified by peer review) is the author/funder, who has granted medRxiv a license to display the preprint in perpetuity.

It is made available under a CC-BY 4.0 International license.

Limited data were available for our study and further research is needed to explain mitigation behavior. The CIS may have sampling bias and the questionnaire did not support the computation of previously validated psychological scores. The anxiety-depression-stress scale may only be suitable for this epidemiologic study. Lastly, we only validated scale reliability using Chronbach’s alpha.

\section{References}

1. Abigail Wozniak, Joe Willey, Jennifer Benz, and Nick Hart. COVID Impact Survey. Chicago, IL: National Opinion Research Center, 2020.

2. Abigail Wozniak. Disparities and Mitigation Behavior during COVID-19. Opportunity \& Inclusive Growth Institute Working Paper 32, 2020. DOI: https://doi.org/10.21034/iwp.32

3. CDC Coronavirus Disease 2019 (COVID-19). People Who Are At Higher Risk. https://www.cdc.gov/coronavirus/2019-ncov/need-extra-precautions/groups-at-higher-risk.html Accessed June 13, 2020.

4. BRFSS Survey Data. Centers for Disease Control and Prevention (CDC). Behavioral Risk Factor Surveillance System Survey Data. Atlanta, Georgia: U.S. Department of Health and Human Services, Centers for Disease Control and Prevention, 2018.

https://www.cdc.gov/brfss/annual_data/annual_data.htm

Accessed June 13, 2020. 
medRxiv preprint doi: https://doi.org/10.1101/2020.07.20.20157925; this version posted July 25, 2020. The copyright holder for this preprint (which was not certified by peer review) is the author/funder, who has granted medRxiv a license to display the preprint in perpetuity.

It is made available under a CC-BY 4.0 International license .

Table 1. Descriptive Statistic for Mitigation Compliance and Anxiety-Depression-Stress Scales and Computed Risk Factors and Symptom Scores based on the COVID Impact Survey (CIS) in 2020

\begin{tabular}{|c|c|c|c|}
\hline & $\begin{array}{r}\text { CIS Wave 1: } \\
\text { April 20-26, } 2020 \\
\end{array}$ & $\begin{array}{r}\text { CIS Wave 2: } \\
\text { May 4-10, } 2020 \\
\end{array}$ & $\begin{array}{r}\text { CIS Wave 3: } \\
\text { May 30- June 8, } 2020\end{array}$ \\
\hline \multicolumn{4}{|l|}{ Mitigation Compliance Scale } \\
\hline Reliability Coefficient & 0.724 & 0.726 & 0.803 \\
\hline Mean & 8.20 & 7.96 & 7.73 \\
\hline Std. Deviation & 3.26 & 3.27 & 3.48 \\
\hline Median & 8 & 8 & 8 \\
\hline Range & $0-17$ & $0-17$ & $0-17$ \\
\hline Low (0 to 6$)$ & $28.9 \%$ & $31.3 \%$ & $33.3 \%$ \\
\hline Moderate (7 to 9 ) & $35.8 \%$ & $34.5 \%$ & $35.2 \%$ \\
\hline High (10 to 17$)$ & $35.3 \%$ & $34.2 \%$ & $31.5 \%$ \\
\hline \multicolumn{4}{|l|}{ Anxiety-Depression-Stress Scale } \\
\hline Reliability Coefficient & 0.821 & 0.815 & 0.857 \\
\hline Mean & 7.80 & 7.44 & 7.71 \\
\hline Std. Deviation & 3.41 & 3.08 & 3.45 \\
\hline Median & 7 & 6 & 6 \\
\hline Range & $5-20$ & $5-20$ & $5-20$ \\
\hline Low (5) & $36.6 \%$ & $39.5 \%$ & $39.0 \%$ \\
\hline Moderate (6 to 8) & $33.6 \%$ & $33.9 \%$ & $32.9 \%$ \\
\hline High (9 to 20) & $29.8 \%$ & $26.6 \%$ & $28.1 \%$ \\
\hline \multicolumn{4}{|l|}{ Risk Factors Score } \\
\hline Mean & 0.98 & 0.99 & 0.90 \\
\hline Std. Deviation & 1.24 & 1.18 & 1.21 \\
\hline Median & 1 & 1 & 1 \\
\hline Range & $0-9$ & $0-9$ & $0-9$ \\
\hline No risk factors & $52.4 \%$ & $50.5 \%$ & $54.8 \%$ \\
\hline 1 or more risk factors & $47.6 \%$ & $49.5 \%$ & $45.2 \%$ \\
\hline \multicolumn{4}{|l|}{ Symptoms Score } \\
\hline Mean & 1.16 & 1.07 & 1.01 \\
\hline Std. Deviation & 1.41 & 1.38 & 1.30 \\
\hline Median & 1 & 1 & 1 \\
\hline Range & $0-8$ & $0-8$ & $0-8$ \\
\hline No symptoms & $47.9 \%$ & $50.9 \%$ & $51.5 \%$ \\
\hline 1 or 2 symptoms & $36.9 \%$ & $35.5 \%$ & $36.6 \%$ \\
\hline 3 or more symptoms & $15.2 \%$ & $13.6 \%$ & $11.9 \%$ \\
\hline
\end{tabular}


Table 2. Multivariate Regressions with Mitigation Compliance Scale as the Dependent Variable and 4 Sets of Independent Variables, COVID Impact Survey (CIS) 2020.

\begin{tabular}{|c|c|c|c|c|c|c|c|c|c|c|c|c|}
\hline \multirow[b]{2}{*}{$\begin{array}{l}\text { Independent } \\
\text { Variables }\end{array}$} & \multicolumn{4}{|c|}{ CIS Wave 1: April 20-26, 2020} & \multicolumn{4}{|c|}{ CIS Wave 2: May 4-10, 2020} & \multicolumn{4}{|c|}{ CIS Wave 3: May 30- June 8, 2020} \\
\hline & Model 1 & Model 2 & Model 3 & Model 4 & Model 1 & Model 2 & Model 3 & Model 4 & Model 1 & Model 2 & Model 3 & Model 4 \\
\hline Symptoms Score & $\begin{array}{r}.194 \\
(.050) \\
<.001\end{array}$ & $\begin{array}{r}.145 \\
(.052) \\
.005\end{array}$ & $\begin{array}{r}.135 \\
(.051) \\
.007\end{array}$ & $\begin{array}{r}.132 \\
(.050) \\
.009\end{array}$ & $\begin{array}{r}.139 \\
(.052) \\
.008\end{array}$ & $\begin{array}{r}.013 \\
(.052) \\
.803\end{array}$ & $\begin{array}{r}.028 \\
(.051) \\
.588\end{array}$ & $\begin{array}{r}.036 \\
(.051) \\
.481\end{array}$ & $\begin{array}{r}.201 \\
(.061) \\
.001\end{array}$ & $\begin{array}{r}.079 \\
(.062) \\
.199\end{array}$ & $\begin{array}{r}.084 \\
(.060) \\
.159\end{array}$ & $\begin{array}{r}.108 \\
(.059) \\
.071\end{array}$ \\
\hline Risk Factors Score & $\begin{array}{r}-.006 \\
(.058) \\
.919 \\
\end{array}$ & $\begin{array}{r}-.008 \\
(.060) \\
.894 \\
\end{array}$ & $\begin{array}{r}.028 \\
(.059) \\
.637 \\
\end{array}$ & $\begin{array}{r}.051 \\
(.058) \\
.383 \\
\end{array}$ & $\begin{array}{r}.077 \\
(.060) \\
.201 \\
\end{array}$ & $\begin{array}{r}.074 \\
(.061) \\
.226 \\
\end{array}$ & $\begin{array}{r}.142 \\
(.060) \\
.018 \\
\end{array}$ & $\begin{array}{r}.163 \\
(.060) \\
.006 \\
\end{array}$ & $\begin{array}{r}-.069 \\
(.067) \\
.303 \\
\end{array}$ & $\begin{array}{r}-.089 \\
(.068) \\
.194 \\
\end{array}$ & $\begin{array}{r}-.064 \\
(.066) \\
.337 \\
\end{array}$ & $\begin{array}{r}-.015 \\
(.066) \\
.816\end{array}$ \\
\hline Age 65 or older & & $\begin{array}{r}-.043 \\
(.177) \\
.809\end{array}$ & $\begin{array}{r}.113 \\
(.174) \\
.515\end{array}$ & $\begin{array}{r}.163 \\
(.173) \\
.347 \\
\end{array}$ & & $\begin{array}{r}.076 \\
(.170) \\
.654 \\
\end{array}$ & $\begin{array}{r}.237 \\
(.167) \\
.156 \\
\end{array}$ & $\begin{array}{r}.292 \\
(.166) \\
.080 \\
\end{array}$ & & $\begin{array}{r}.209 \\
(.196) \\
.288\end{array}$ & $\begin{array}{r}.238 \\
(.193) \\
.218 \\
\end{array}$ & $\begin{array}{r}.305 \\
(.191) \\
.111\end{array}$ \\
\hline $\begin{array}{l}\text { Anxiety- } \\
\text { Depression-Stress } \\
\text { Scale }\end{array}$ & & $\begin{array}{r}.082 \\
(.021) \\
<.001 \\
\end{array}$ & $\begin{array}{r}.081 \\
(.021) \\
<.001 \\
\end{array}$ & $\begin{array}{r}.091 \\
(.021) \\
<.001 \\
\end{array}$ & & $\begin{array}{r}.230 \\
(.023) \\
<.001 \\
\end{array}$ & $\begin{array}{l}.281 \\
(.023) \\
<.001 \\
\end{array}$ & $\begin{array}{r}.217 \\
(.023) \\
<.001 \\
\end{array}$ & & $\begin{array}{r}.189 \\
(.023) \\
<.001 \\
\end{array}$ & $\begin{array}{l}.182 \\
(.022) \\
<.001 \\
\end{array}$ & $\begin{array}{r}.191 \\
(.022) \\
<.001 \\
\end{array}$ \\
\hline Female & & & $\begin{array}{r}.784 \\
(.136) \\
<.001 \\
\end{array}$ & $\begin{array}{r}.879 \\
(.135) \\
<.001 \\
\end{array}$ & & & $\begin{array}{r}.671 \\
(.134) \\
<.001 \\
\end{array}$ & $\begin{array}{r}.762 \\
(.135) \\
<.001 \\
\end{array}$ & & & $\begin{array}{r}.867 \\
(.148) \\
<.001 \\
\end{array}$ & $\begin{array}{r}.968 \\
(.147) \\
<.001 \\
\end{array}$ \\
\hline Non-White & & & $\begin{array}{r}.574 \\
(.142) \\
<.001 \\
\end{array}$ & $\begin{array}{r}.652 \\
(.146) \\
<.001 \\
\end{array}$ & & & $\begin{array}{r}.528 \\
(.141) \\
<.001 \\
\end{array}$ & $\begin{array}{r}.505 \\
(.145) \\
<.001 \\
\end{array}$ & & & $\begin{array}{r}.232 \\
(.154) \\
.133 \\
\end{array}$ & $\begin{array}{r}.244 \\
(.158) \\
.124\end{array}$ \\
\hline $\begin{array}{l}\text { Post-Secondary } \\
\text { Degree }\end{array}$ & & & $\begin{array}{l}1.215 \\
(.137) \\
<.001\end{array}$ & $\begin{array}{r}.927 \\
(.142) \\
<.001\end{array}$ & & & $\begin{array}{l}1.165 \\
(.134) \\
<.001\end{array}$ & $\begin{array}{r}.960 \\
(.139) \\
<.001\end{array}$ & & & $\begin{array}{l}1.362 \\
(.148) \\
<.001\end{array}$ & $\begin{array}{l}1.060 \\
(.153) \\
<.001\end{array}$ \\
\hline Urban Location & & & & $\begin{array}{r}.409 \\
(.156) \\
.009\end{array}$ & & & & $\begin{array}{r}.439 \\
(.153) \\
.004 \\
\end{array}$ & & & & $\begin{array}{r}.613 \\
(.167) \\
<.001\end{array}$ \\
\hline $\begin{array}{l}\text { Household income } \\
\text { greater than } \\
\$ 50,000 \text { annually }\end{array}$ & & & & $\begin{array}{r}.860 \\
(.147) \\
<.001 \\
\end{array}$ & & & & $\begin{array}{r}.615 \\
(.142) \\
<.001 \\
\end{array}$ & & & & $\begin{array}{r}.902 \\
(.156) \\
<.001\end{array}$ \\
\hline $\begin{array}{l}\text { Household member } \\
\text { with COVID-19 } \\
\text { diagnosis }\end{array}$ & & & & $\begin{array}{r}.897 \\
(.690) \\
.193\end{array}$ & & & & $\begin{array}{r}.867 \\
(.616) \\
.159\end{array}$ & & & & $\begin{array}{r}-1.528 \\
(.556) \\
.006\end{array}$ \\
\hline Adjusted R-squared & .009 & .012 & .064 & .081 & .004 & .046 & .089 & .101 & .004 & .036 & .090 & .113 \\
\hline
\end{tabular}


Notes: Cell entries are regression coefficient B, (SE), and P-value.

Mitigation compliance scale summed 17 items: washing or sanitizing hands, wiping packages entering the home, wearing face masks, staying at home when unwell, keeping distance from individuals outside the household, postponing or cancelling doctor appointments, dental appointments, housekeeper or caregiver services, recreational activities, work activities, school activities, avoiding going to restaurants, public or crowded places, avoiding contact with high-risk people, stockpiling of food or water, working from home, and studying from home. Scale reliability was $0.72-0.80$.

Anxiety-depression-stress scale summed days of feelings of depression, anxiety, hopelessness, and loneliness, and stress experienced by CIS respondents within the last 7 days. Scale reliability was $0.81-0.86$.

Symptoms score summed fever, chills, shortness of breath (dyspnea), cough, sore throat, fatigue or tiredness, muscle pain (myalgia), and loss of taste and smell (anosmia).

Risk factors score summed diabetes, high blood pressure or hypertension, heart disease or stroke, asthma, chronic lung disease or COPD, bronchitis or emphysema, liver disease, cancer, and immunocompromised condition. 